\title{
Homogeneity and non-coincidence of Hausdorff and box dimensions for subsets of $\mathbb{R}^{n}$
}

\author{
by
}

\author{
Anders Nilsson and Peter Wingren (Umeå)
}

\begin{abstract}
A class of subsets of $\mathbb{R}^{n}$ is constructed that have certain homogeneity and non-coincidence properties with respect to Hausdorff and box dimensions. For each triple $(r, s, t)$ of numbers in the interval $(0, n]$ with $r<s<t$, a compact set $K$ is constructed so that for any non-empty subset $U$ relatively open in $K$, we have $\left(\operatorname{dim}_{\mathrm{H}}(U), \underline{\operatorname{dim}}_{\mathrm{B}}(U), \overline{\operatorname{dim}}_{\mathrm{B}}(U)\right)=(r, s, t)$. Moreover, $2^{-n} \leq H^{r}(K) \leq 2 n^{r / 2}$.
\end{abstract}

1. Introduction and result. In this work we present a general construction of Cantor type. It produces compact sets that have certain homogeneity properties and non-coinciding dimensions with respect to Hausdorff, lower box and upper box dimensions. The result is formulated in the theorem below. It says that for any given numbers $r, s, t$ in $(0, n]$ with $r<s<t$, there is a compact set $K$ in $\mathbb{R}^{n}$ such that the local dimensions equal the global dimensions in the following sense. For any subset $U$ relatively open in $K$, we have

$$
\left(\operatorname{dim}_{\mathrm{H}}(U), \underline{\operatorname{dim}}_{\mathrm{B}}(U), \overline{\operatorname{dim}}_{\mathrm{B}}(U)\right)=(r, s, t) .
$$

It is well known, and easily seen from the definitions, that

$$
\operatorname{dim}_{\mathrm{H}}(U) \leq \underline{\operatorname{dim}}_{\mathrm{B}}(U) \leq \overline{\operatorname{dim}}_{\mathrm{B}}(U) .
$$

In the literature there are many examples and constructions of classes of compact sets $K$ for which one or both inequalities in (1) are strict; see e.g. [3], [2], [4], [5]. The backgrounds and directions of these constructions vary. Since our goal is to establish a class of compact sets that have the above homogeneity and non-coincidence property, it is natural to compare with [4] and [5]. We believe that this comparison will throw some light on the position of the present work in this area.

2000 Mathematics Subject Classification: 28A78, 28A80.

Key words and phrases: fractal, Cantor set construction, homogeneous, Hausdorff dimension, box dimension, packing dimension.

Research of P. Wingren was supported by the Swedish Research Council (grant 6212002-3795). 
Our result could be seen as a generalization of an elegant construction by Pesin and Weiss [4, pp. 129-132]. Their construction allows room for variation, and it is possible, within that scope, to produce sets that satisfy the special homogeneity property. The special blend of different features in our construction of $K$ allows us to extend the result in [4] from compact sets $K$ in $\mathbb{R}^{n}, n=2$ with $r, s, t$ in $(0, \log 2 / \log 3)$ to compact sets $K$ in $\mathbb{R}^{n}$ for $n$ arbitrary and with $r, s, t$ in $(0, n]$. The cost of the generalization is some loss of elegance.

As an example of a more loosely related result, we mention the interesting result of [5]. In that work, a construction of a compact subset of $[0,1]$ is presented where a coutable number of small disjoint subintervals of $[0,1]$ are chosen from right to left. Each of these intervals contains a subset $Y_{l}$ with $\operatorname{dim}_{H}\left(Y_{l}\right)=s$ and $\operatorname{dim}_{\mathrm{P}}\left(Y_{l}\right)=t, l=1,2, \ldots$ The union $\bigcup Y_{l}$ has the same dimensions as $Y_{l}$ due to countable stability of Hausdorff and packing dimensions. The lack of countable stability of box dimensions makes it possible to arrange the $Y_{l}$-sets when going from right to left in such a way that the box dimensions deviate from the Hausdorff and packing dimensions. One way to investigate the homogeneity and non-coincidence properties of the compact sets in [4] and the present paper on one hand, and of the compact sets in [5] on the other hand, is to study the function

$D(x)=\lim _{\delta \rightarrow 0}\left(\operatorname{dim}_{\mathrm{H}}\left(U_{\delta}(x)\right), \underline{\operatorname{dim}}_{\mathrm{B}}\left(U_{\delta}(x)\right), \overline{\operatorname{dim}}_{\mathrm{B}}\left(U_{\delta}(x)\right), \operatorname{dim}_{\mathrm{P}}\left(U_{\delta}(x)\right)\right), x \in K$, where $U_{\delta}(x)=B_{\delta}(x) \cap K$. For $K$ in [5], $0<r<s<t<1$ and $r<u<t$,

$$
D(0)=(r, s, t, u) \quad \text { and } \quad D(x)=(s, s, t, t), x \in K \backslash\{0\} .
$$

Thus, non-coincidence with respect to all of the four studied dimensions holds locally only at the point $x=0$. At all other $x$-values in $K$, the Hausdorff dimension and the lower box dimension coincide, as also do the upper box dimension and the packing dimension. However, for [4] and for our result,

$$
D(x)=(r, s, t, t) \quad \text { if } x \in K .
$$

Note that simultaneous homogeneity and non-coincidence is impossible to reach for the upper box and packing dimension (see e.g. [1, p. 49]). Thus, the following theorem is sharp.

Theorem. Given $r, s, t \in(0, n], r<s<t$, there is a compact set $K \subset \mathbb{R}^{n}$ such that for each non-empty set $U$ relatively open in $K$,

$$
\begin{aligned}
& \overline{\operatorname{dim}}_{\mathrm{B}}(U)=t, \\
& \underline{\operatorname{dim}}_{\mathrm{B}}(U)=s, \\
& \operatorname{dim}_{\mathrm{H}}(U)=r .
\end{aligned}
$$


REMARK 1. The proof of (4) includes the distinct result that the $r$ dimensional Hausdorff measure of $K$ is in the interval $\left[2^{-n}, 2 n^{r / 2}\right]$. It is also possible to see from the proof that $0<H^{r}(U)<\infty$ for each non-empty set $U$ relatively open in $K$.

REMARK 2. With a minor adjustment in the construction, it is possible to allow $r=0$, but in this case the $r$-dimensional Hausdorff measure will be infinite, i.e. $H^{r}(K)=\infty$.

2. Construction features that facilitate the proof. We list these features as (i) $-(\mathrm{v})$ below.

(i) The set $K$ is constructed by a Cantor set procedure. It is the intersection of a decreasing sequence of compact sets, i.e. $K=\bigcap_{i=0}^{\infty} K_{i}$ where $K_{i} \supset K_{i+1}$ for all $i$. Each $K_{i}$ is a finite union of closed dyadic cubes of side length $2^{-i}$.

(ii) For each cube in such a compact union, at least one of its dyadic subcubes of half the side length is chosen to be part of the next step (i.e. union) in the construction. Moreover, for an infinite number of $i$, we have $K_{i}=K_{i+1}$. Hence, each cube in the construction has an interior point that belongs to $K$. We say that the cubes in the construction have the non-empty interior property with respect to $K$. This can be used to prove the following lemma.

Lemma. Let $p_{1}<p_{2} \leq q$ be positive integers. Let $\mathcal{F}$ be a collection of closed dyadic cubes with side lengths in the interval $\left[2^{-p_{2}}, 2^{-p_{1}}\right]$. Then $\mathcal{F}$ is a cover of $K$ if and only if $\mathcal{F}$ is a cover of $K_{q}$.

Proof. The sufficiency is obvious, so assume that $\mathcal{F}$ is a cover of $K$. Take an arbitrary closed dyadic cube $Q \subset K_{q}$ of side length $2^{-q}$. The interior of $Q$ must contain at least one point $x \in K$ according to the non-empty interior property with respect to $K$. Since $\mathcal{F}$ is a cover of $K$, there exists $Q^{*} \in \mathcal{F}$ that contains $x$. The only possible closed dyadic cubes that contain interior points of $Q$ are either subcubes of $Q$ or cubes that contain $Q$. Since the side length of $Q$ is less than or equal to the side length of $Q^{*}$, we must have $Q \subset Q^{*}$. Hence, $\mathcal{F}$ is a cover of $K_{q}$.

This property helps to control the number of dyadic cubes of a certain side length that intersect the compact set $K$, and hence to reach the right dimension properties.

(iii) The upper and lower box dimensions of $K$ are determined by the sequence

$$
\left\{\frac{\log n_{k}(K)}{-\log 2^{-k}}\right\}_{k=1}^{\infty}
$$


where $n_{k}(K)$ is the number of dyadic cubes with side length $2^{-k}$ that intersect $K$. As a consequence, it is suitable to begin the construction by defining sequences of integers with accumulation points $t$ and $s$. To achieve (4), these sequences should also have an accumulation point at $r$. We find it convenient to define two sequences with greatest and smallest accumulation points $t$ and $r$, and coordinate them with respect to $s$. By using these sequences to choose cubes according to (i) and (ii), we pave the way for the proof of (2)-(4).

(iv) To make the calculations more transparent, we pick the number of subcubes of a given cube to be a positive integer power of 2. Consequently, we are able to write the general element in (5) as an arithmetical mean.

(v) This fifth and last feature is important for the proof of (4). Given a positive integer $m$, the cubes in the construction with side lengths $2^{-m}$ are members of two special families. In each of these families all cubes have the same future with respect to offspring subcubes, which may be different from the future of the cubes in the other family, seen from a short term perspective. However, in the long run, their futures are eventually the same. The words the same future summarize what can only be understood from the detailed proof and roughly speaking mean have the same number of offspring subcubes.

3. Proof of the theorem. The proof is divided into three parts. In part I, we construct two sequences of integers with some special properties. In part II, we use them to construct two sequences of families of cubes in $\mathbb{R}^{n}$. The latter define a decreasing sequence of non-empty compact sets in $\mathbb{R}^{n}$. The set $K$ is then defined to be the non-empty compact intersection of this decreasing sequence. In part III, we prove that $K$ has properties (2)-(4).

Proof. PART I. We define two sequences of integers named the 0 -sequence and the 1 -sequence, $\left\{\alpha^{(j)}(i)\right\}_{i=1}^{\infty}, j=0,1$, where $\alpha^{(j)}(i) \in\{0, n\}$. The values in these sequences are chosen consecutively, and each value depends on the previous ones. In this process, the arithmetic means

$$
\bar{\alpha}^{(j)}(k)=\frac{1}{k} \sum_{i=1}^{k} \alpha^{(j)}(i), \quad k=1,2, \ldots, j=0,1,
$$

play a role, as also does $\varepsilon$ defined by $\varepsilon=\frac{1}{2} \min \{t-s, s-r\}$. In each step, we choose one element per sequence. The choices are made according to a special rule, and the rules are repeated periodically. The steps included in each such period are called a multistep. Multistep $p$ starts with step $\sigma_{p-1}+1$ and ends with step $\sigma_{p}$, where $\left\{\sigma_{p}\right\}_{p=1}^{\infty}$ is an increasing sequence of integers 
such that

$$
\sigma_{p} \geq n p(p+1) / \varepsilon \text {. }
$$

These numbers will be precisely defined later on. Thus, Multistep 1 consists of steps $i=1, \ldots, \sigma_{1}$ corresponding to choosing $\alpha^{(0)}(i)$ and $\alpha^{(1)}(i)$. Multistep $p>1$ corresponds to choosing $\alpha^{(0)}(i)$ and $\alpha^{(1)}(i)$ for $i=\sigma_{p-1}+1$, $\sigma_{p-1}+2, \ldots, \sigma_{p}$. By describing how to choose elements in Multistep 1, and in Multistep $p+1$ given that Multistep $p \geq 1$ is finished, the two sequences are completely defined.

We now describe the successive choice of elements in the 0-sequence and 1-sequence. By introducing a special auxiliary sequence, we make it easier to choose elements so that the sequences get the right properties. The auxiliary sequence $\left\{c_{i}\right\}_{i=1}^{\infty}$ is defined by

$$
c_{i}= \begin{cases}0 & \text { if } i=1 \\ n H\left(s-\bar{c}_{i-1}\right) & \text { if } i>1\end{cases}
$$

where $H(x)$ is the Heaviside function, i.e. 0 for negative $x$-values and 1 for nonnegative ones, and

$$
\bar{c}_{i}=\frac{1}{i} \sum_{j=1}^{i} c_{j} .
$$

Later we will use the property $\left|s-\bar{c}_{i}\right| \leq n / i$ for all $i$. This can easily be shown by induction, but we omit the simple proof. Let $S=\left\{i: c_{i+1}=n\right\}$. This set is unbounded since $s>0$. In the process below, all $\sigma_{p}$ will belong to $S$.

Now, we begin with Multistep 1 , and we choose $\sigma_{1} \in S$ so that (6) holds. This is possible because $S$ is unbounded. For $i=1, \ldots, \sigma_{1}$, choose $\alpha^{(0)}(i)=\alpha^{(1)}(i)=c_{i}$. This gives us $\bar{\alpha}^{(0)}\left(\sigma_{1}\right)=\bar{\alpha}^{(1)}\left(\sigma_{1}\right)=\bar{c}_{\sigma_{1}}$, hence

$$
\left|s-\bar{\alpha}^{(j)}\left(\sigma_{1}\right)\right| \leq n / \sigma_{1}, \quad j=0,1 .
$$

Now, suppose that Multistep $p \geq 1$ is finished, so we have chosen values for $i=1, \ldots, \sigma_{p}$. Furthermore, suppose that we have the starting conditions $\bar{\alpha}^{(0)}\left(\sigma_{p}\right)=\bar{\alpha}^{(1)}\left(\sigma_{p}\right)=\bar{c}_{\sigma_{p}}$ so that

$$
\left|s-\bar{\alpha}^{(j)}\left(\sigma_{p}\right)\right| \leq n / \sigma_{p}, \quad j=0,1,
$$

where $\sigma_{p} \in S$ and (6) holds. Hence, the starting conditions regulate the distances between $s$ and the last mean values in Multistep $p$. We will now describe Multistep $p+1$.

The elements in the 1 -sequence are chosen for $i=\sigma_{p}+1, \ldots, \sigma_{p+1}$ in the following way. For $i>\sigma_{p}$, let $\alpha^{(1)}(i)=n$ until $\bar{\alpha}^{(1)}(i) \geq t-\varepsilon / p$, which must occur for some integer $i=i_{1}$ because the mean values $\bar{\alpha}^{(1)}(i)$ will increase towards $n$ and $t-\varepsilon / p<n$. Furthermore, the definition of $\varepsilon$ together with (6) implies that $\bar{\alpha}^{(1)}\left(\sigma_{p}\right)<t-\varepsilon / p$, so at least one value must be chosen in 
this way before the inequality is satisfied. Then, for $i>i_{1}$, let $\alpha^{(1)}(i)=0$ until $\bar{\alpha}^{(1)}(i)<r$, which must occur for some integer $i=i_{2}$ because the mean values $\bar{\alpha}^{(1)}(i)$ will decrease towards 0 and $r>0$. At least one value must be chosen in this way before the inequality is fulfilled, by the same reasoning as above. Now, for $i>i_{2}$, let $\alpha^{(1)}(i)=n$ until $\bar{\alpha}^{(1)}(i)=\bar{c}_{i}$. This must occur for some integer $i=i_{3}$ because the function $i \mapsto i \bar{c}_{i}-i \bar{\alpha}^{(1)}(i)$ is a positive integer multiple of $n$ for $i=i_{2}$. For $i>i_{2}$, it decreases by 0 or $n$ in each step. Since $c_{i}=0$ for an infinite number of $i$, the function reaches 0 when $i$ is large enough. Finally, for $i>i_{3}$, let $\alpha^{(1)}(i)=c_{i}$ until $i=\sigma_{p+1}$, which will be defined later.

The elements in the 0 -sequence are chosen for $i=\sigma_{p}+1, \ldots, \sigma_{p+1}$ in the following way. For $i>\sigma_{p}$, let $\alpha^{(0)}(i)=c_{i}$ until $i=i_{3}$, and then for $i>i_{3}$, let $\alpha^{(0)}(i)=n$ until $\bar{\alpha}^{(0)}(i) \geq t-\varepsilon / p$ for some integer $i=i_{4}$. Now, for $i>i_{4}$, let $\alpha^{(0)}(i)=0$ until $\bar{\alpha}^{(0)}(i)<r$ for some integer $i=i_{5}$, and for $i>i_{5}$, let $\alpha^{(0)}(i)=n$ until $\bar{\alpha}^{(0)}(i)=\bar{c}_{i}$ for some integer $i=i_{6}$. For $i>i_{6}$, let $\alpha^{(0)}(i)=c_{i}$ until $i=\sigma_{p+1}$, which we can now define as an arbitrary integer in $S$ larger than $\max \left\{i_{6}, n(p+1)(p+2) / \varepsilon\right\}$. Note that the values $i_{l}$, $l=1, \ldots, 6$, depend on $p$, but since this is obvious from the context we omit it in the notation. Note, moreover, that for $i=i_{6}, i_{6}+1, \ldots, \sigma_{p+1}$ we have $\bar{\alpha}^{(0)}(i)=\bar{\alpha}^{(1)}(i)=\bar{c}_{i}$. Consequently,

$$
\left|s-\bar{\alpha}^{(j)}\left(\sigma_{p+1}\right)\right| \leq n / \sigma_{p+1}, \quad j=0,1 .
$$

This together with the choice of $\sigma_{p+1}$ implies that the starting conditions for Multistep $p+2$ are satisfied. Induction now gives us the complete 0 - and 1 -sequences.

The 0 -sequence and the 1-sequence defined above have the following properties that we will use later. For the 1-sequence, we have

$$
\begin{aligned}
& \max \left\{\bar{\alpha}^{(1)}(i): i=\sigma_{p}, \sigma_{p}+1, \ldots, \sigma_{p+1}\right\}=\bar{\alpha}^{(1)}\left(i_{1}\right) \in\left[t-\frac{\varepsilon}{p}, t-\frac{\varepsilon}{p+1}\right), \\
& \min \left\{\bar{\alpha}^{(1)}(i): i=\sigma_{p}, \sigma_{p}+1, \ldots, \sigma_{p+1}\right\}=\bar{\alpha}^{(1)}\left(i_{2}\right) \in\left[r-\frac{n}{i_{2}}, r\right)
\end{aligned}
$$

and

$$
\left|s-\bar{\alpha}^{(1)}(i)\right| \leq n / i, \quad i=\sigma_{p} \text { and } i=i_{3}, i_{3}+1, \ldots, \sigma_{p+1} .
$$

For the 0 -sequence, we have

$$
\begin{aligned}
& \max \left\{\bar{\alpha}^{(0)}(i): i=\sigma_{p}, \sigma_{p}+1, \ldots, \sigma_{p+1}\right\}=\bar{\alpha}^{(0)}\left(i_{4}\right) \in\left[t-\frac{\varepsilon}{p}, t-\frac{\varepsilon}{p+1}\right) \\
& \min \left\{\bar{\alpha}^{(0)}(i): i=\sigma_{p}, \sigma_{p}+1, \ldots, \sigma_{p+1}\right\}=\bar{\alpha}^{(0)}\left(i_{5}\right) \in\left[r-\frac{n}{i_{5}}, r\right)
\end{aligned}
$$


and

(12) $\left|s-\bar{\alpha}^{(0)}(i)\right| \leq n / i, \quad i=\sigma_{p}, \sigma_{p}+1, \ldots, i_{3}$ and $i=i_{6}, i_{6}+1, \ldots, \sigma_{p+1}$.

To prove the properties (7)-(12) above, we use

$$
\begin{aligned}
\mid \bar{\alpha}^{(j)}(k)-\bar{\alpha}^{(j)} & (k-1) \mid \\
& =\left|\frac{\bar{\alpha}^{(j)}(k-1) \cdot(k-1)+\alpha^{(j)}(k)}{k}-\frac{\bar{\alpha}^{(j)}(k-1) \cdot k}{k}\right| \\
& =\frac{\left|\alpha^{(j)}(k)-\bar{\alpha}^{(j)}(k-1)\right|}{k} \leq \frac{n}{k} .
\end{aligned}
$$

The equality in (7) is obvious from the choice of $i_{1}$, as also is the relation

$$
\bar{\alpha}^{(1)}\left(i_{1}-1\right)<t-\varepsilon / p \leq \bar{\alpha}^{(1)}\left(i_{1}\right)
$$

This, together with (13) and (6), gives

$$
\begin{aligned}
\bar{\alpha}^{(1)}\left(i_{1}\right) & =\bar{\alpha}^{(1)}\left(i_{1}\right)-\bar{\alpha}^{(1)}\left(i_{1}-1\right)+\bar{\alpha}^{(1)}\left(i_{1}-1\right)<\frac{n}{i_{1}}+t-\frac{\varepsilon}{p} \\
& \leq t-\left(\frac{\varepsilon}{p}-\frac{n}{\sigma_{p}}\right) \leq t-\varepsilon\left(\frac{1}{p}-\frac{1}{p(p+1)}\right)=t-\frac{\varepsilon}{p+1} .
\end{aligned}
$$

Hence, (7) is proved. Property (10) can be proved in the same way. Similarly, it is obvious that the equality in (8) follows from the choice of $i_{2}$, as does the relation

$$
\bar{\alpha}^{(1)}\left(i_{2}-1\right) \geq r>\bar{\alpha}^{(1)}\left(i_{2}\right) .
$$

This, together with (13) and (6), gives

$$
\bar{\alpha}^{(1)}\left(i_{2}\right)=\bar{\alpha}^{(1)}\left(i_{2}-1\right)-\left(\bar{\alpha}^{(1)}\left(i_{2}-1\right)-\bar{\alpha}^{(1)}\left(i_{2}\right)\right) \geq r-n / i_{2} .
$$

Hence, (8) is proved. Property (11) can be proved in the same way. Property (9) follows from the fact that for $i=\sigma_{p}$ and $i=i_{3}, i_{3}+1, \ldots, \sigma_{p+1}$ we have $\bar{\alpha}^{(1)}(i)=\bar{c}_{i}$. Similarly, property (12) follows from the fact that for $i=\sigma_{p}, \sigma_{p}+1, \ldots, i_{3}$ and $i=i_{6}, i_{6}+1, \ldots, \sigma_{p+1}$ we have $\bar{\alpha}^{(0)}(i)=\bar{c}_{i}$.

PART II. We will now define two sequences $\left\{\mathcal{F}^{(j)}(i)\right\}_{i=0}^{\infty}, j=0,1$, where each element $\mathcal{F}^{(j)}(i)$ is a non-empty family of closed cubes in $\mathbb{R}^{n}$. The union of the cubes in $\mathcal{F}^{(0)}(i) \cup \mathcal{F}^{(1)}(i)$ is a non-empty compact subset of $\mathbb{R}^{n}$, and the families are chosen so that the corresponding sequence of compact sets is decreasing. The set $K$ is then defined to be the non-empty compact intersection of the compact sets in this decreasing sequence.

The families of cubes are defined in a procedure similar to that described for the 0 -sequence and the 1-sequence in part I above. Each family of cubes $\mathcal{F}^{(j)}(i)$ is a subset of $\mathcal{F}(i)$ defined by

$$
\mathcal{F}(i)= \begin{cases}\left\{\left[m_{1}, m_{1}+1\right] \times \cdots \times\left[m_{n}, m_{n}+1\right]: m_{1}, \ldots, m_{n} \in \mathbb{Z}\right\} & \text { if } i=0 \\ \left\{Q \subset \mathbb{R}^{n}: 2^{i} \cdot Q \in \mathcal{F}(0)\right\} & \text { if } i>0\end{cases}
$$


Note that each cube in $\mathcal{F}(i)$ has exactly $2^{n}$ subcubes in $\mathcal{F}(i+1)$. Now, we begin the process of choosing families. Let $\mathcal{F}^{(j)}(0)=\left\{[0,1]^{n}+(j, 0, \ldots, 0)\right\}$ for $j=0,1$. Multistep 1 consists of choosing families for $i=1, \ldots, \sigma_{1}$. Suppose the family $\mathcal{F}^{(j)}(i-1)$ has been chosen for some $i \geq 1$, and we are about to choose $\mathcal{F}^{(j)}(i)$. Each cube in $\mathcal{F}^{(j)}(i-1)$ has $2^{n}$ subcubes in $\mathcal{F}(i)$. Let $2^{\alpha^{(j)}(i)}$ of these belong to $\mathcal{F}^{(j)}(i)$. Thus, the number of cubes in $\mathcal{F}^{(j)}(i)$ is $2^{\alpha^{(j)}(i)}$ times the number of cubes in $\mathcal{F}^{(j)}(i-1)$. This gives us the families in Multistep 1, and for $i=1, \ldots, \sigma_{1}$, the number of cubes in $\mathcal{F}^{(j)}(i)$ is $2^{\bar{\alpha}^{(j)}(i) \cdot i}, j=0,1$.

Now, suppose that Multistep $p \geq 1$ is finished, so that we have chosen families for $i=0,1, \ldots, \sigma_{p}$. We will now describe Multistep $p+1$. The families $\mathcal{F}^{(0)}\left(\sigma_{p}\right)$ and $\mathcal{F}^{(1)}\left(\sigma_{p}\right)$ have the same number of cubes since $\bar{\alpha}^{(0)}\left(\sigma_{p}\right)=\bar{\alpha}^{(1)}\left(\sigma_{p}\right)$. Each cube in $\mathcal{F}^{(0)}\left(\sigma_{p}\right) \cup \mathcal{F}^{(1)}\left(\sigma_{p}\right)$ has $2^{n}$ subcubes in $\mathcal{F}\left(\sigma_{p}+1\right)$. Let $2^{n-1}$ of them belong to $\mathcal{F}^{(0)}\left(\sigma_{p}+1\right)$, and let the other $2^{n-1}$ subcubes belong to $\mathcal{F}^{(1)}\left(\sigma_{p}+1\right)$. Since $\sigma_{p} \in S$, we have $\alpha^{(j)}\left(\sigma_{p}+1\right)=n$ for $j=0,1$, and thus, the number of cubes in $\mathcal{F}^{(j)}\left(\sigma_{p}+1\right)$ is $2^{\alpha^{(j)}\left(\sigma_{p}+1\right)}$ times the number of cubes in $\mathcal{F}^{(j)}\left(\sigma_{p}\right)$.

For $i=\sigma_{p}+2, \ldots, \sigma_{p+1}$, the family $\mathcal{F}^{(j)}(i-1)$ has $2^{n}$ subcubes in $\mathcal{F}(i)$. Let $2^{\alpha^{(j)}(i)}$ of these belong to $\mathcal{F}^{(j)}(i)$. This finishes Multistep $p+1$, and for all $i \leq \sigma_{p+1}$, the number of cubes in $\mathcal{F}^{(j)}(i)$ is given by

$$
\# \mathcal{F}^{(j)}(i)=2^{\bar{\alpha}^{(j)}(i) \cdot i}, \quad j=0,1 .
$$

PART III. Now, we prove the dimension properties (2)-(4) for our set $K$. We begin with (2) and (3) in the case when $U=K$. Let $n_{k}(K)$ be the number of cubes in $\mathcal{F}(k)$ that intersect $K$; hence $n_{k}(K)$ is the number of cubes in $\mathcal{F}^{(0)}(k) \cup \mathcal{F}^{(1)}(k)$ and possibly their closest neighbors from $\mathcal{F}(k)$. By using (14), this can be estimated from below and above as

$$
2^{N(k)} \leq n_{k}(K) \leq 2 \cdot 3^{n} \cdot 2^{N(k)},
$$

where $N(k)=\max \left\{\bar{\alpha}^{(j)}(k) \cdot k: j=0,1\right\}$. This gives

$$
\max _{j=0,1} \bar{\alpha}^{(j)}(k) \leq \frac{\ln n_{k}(K)}{-\ln 2^{-k}} \leq \frac{\ln 2 \cdot 3^{n}}{-\ln 2^{-k}}+\max _{j=0,1} \bar{\alpha}^{(j)}(k) .
$$

By (7) and (10), we have

$$
\varlimsup_{k \rightarrow \infty} \max _{j=0,1} \bar{\alpha}^{(j)}(k)=t
$$

and by (9) and (12), we have

$$
\varliminf_{k \rightarrow \infty} \max _{j=0,1} \bar{\alpha}^{(j)}(k)=s .
$$

This proves (2) and (3) if $U=K$. Now, suppose $U$ is an arbitrary non-empty and relatively open set in $K$, i.e. $U=O \cap K$ for some $O$ open in $\mathbb{R}^{n}$. Then there must exist a $Q \in \mathcal{F}^{(0)}\left(\sigma_{p}\right) \cup \mathcal{F}^{(1)}\left(\sigma_{p}\right)$ for some positive integer $p$ with $Q \subset O$. Consider an arbitrary $k>\sigma_{p}$. We can estimate $n_{k}(Q \cap K)$ from 
below by the number of cubes in $\mathcal{F}^{(0)}(k) \cup \mathcal{F}^{(1)}(k)$ that are subcubes to $Q$. Since all cubes in $\mathcal{F}^{(0)}\left(\sigma_{p}\right) \cup \mathcal{F}^{(1)}\left(\sigma_{p}\right)$ have the same number of subcubes in $\mathcal{F}^{(0)}(k) \cup \mathcal{F}^{(1)}(k)$, and $\# \mathcal{F}^{(0)}\left(\sigma_{p}\right)=\# \mathcal{F}^{(1)}\left(\sigma_{p}\right)$, we can use (14) to make the following estimate:

where

$$
n_{k}(Q \cap K) \geq \frac{\#\left(\mathcal{F}^{(0)}(k) \cup \mathcal{F}^{(1)}(k)\right)}{\#\left(\mathcal{F}^{(0)}\left(\sigma_{p}\right) \cup \mathcal{F}^{(1)}\left(\sigma_{p}\right)\right)}=c\left(2^{\bar{\alpha}^{(0)}(k) \cdot k}+2^{\bar{\alpha}^{(1)}(k) \cdot k}\right),
$$

This gives

$$
c=\frac{1}{\#\left(\mathcal{F}^{(0)}\left(\sigma_{p}\right) \cup \mathcal{F}^{(1)}\left(\sigma_{p}\right)\right)} .
$$

$$
\frac{\ln n_{k}(Q \cap K)}{-\ln 2^{-k}} \geq \frac{\ln c}{-\ln 2^{-k}}+\max _{j=0,1} \bar{\alpha}^{(j)}(k) .
$$

By (7) and (10), we get $\overline{\operatorname{dim}}_{\mathrm{B}}(Q \cap K) \geq t$, and by (9) and (12), it follows that $\underline{\operatorname{dim}}_{\mathrm{B}}(Q \cap K) \geq s$. The upper and lower box dimensions are monotone, and $Q \cap K \subset O \cap K \subset K$, thus (2) and (3) follow.

Now, we prove (4), and we begin with the case $U=K$.

By considering net measures (see for example [1, p. 33]), and since $K$ is compact, it is easy to see that $\operatorname{dim}_{H}(K)$ can be determined by examining finite covers $\left\{Q_{i}\right\}$ of $K$ where each $Q_{i}$ belongs to some $\mathcal{F}^{(j)}(m)$ for a positive integer $m$ and $j \in\{0,1\}$ that depend on $i$.

To show the lower estimate of $(4), \operatorname{dim}_{\mathrm{H}}(K) \geq r$, we consider the class of all families of finite covers of $K$ with cubes from our construction and of side length at most $2^{-\sigma_{p}}$ for some positive integer $p$. We include all such covers in our consideration if we first consider a subclass of covers, namely those where the cubes have side lengths larger than $2^{-\sigma_{\nu}}$ for a $\nu>p$, and then let $\nu \rightarrow \infty$.

Each cover in the above-mentioned subclass is a collection of cubes in our construction, with side lengths in the interval $\left(2^{-\sigma_{\nu}}, 2^{-\sigma_{p}}\right]$. Since there are a finite number of such covers, we may choose a cover, $\mathcal{F}$, that minimizes the Carathéodory sum with respect to $r$. For each positive integer $l$, let $K_{l}=\mathcal{F}^{(0)}(l) \cup \mathcal{F}^{(1)}(l)$.

Choose a largest cube, $Q_{0}$, in the minimizing cover. We have $Q_{0} \in$ $\mathcal{F}^{(j)}(m)$ for some $j=0$ or $j=1$ and some $m \in\left[\sigma_{p}, \sigma_{\nu}\right)$. Then take into account any cube $Q$ in $K_{m}$. We identify $Q$ and its dyadic subcubes of side length not smaller than $2^{-\sigma_{\nu}}$ and appearing in the construction with a finite tree graph in the following way. The cube $Q$ is viewed as the root node. From this root, we have successive edges to nodes that correspond to subcubes of half the side length in the construction. Thus, with this graph-theoretical approach, a finite tree $T(Q)$ is assigned to each cube $Q \in K_{m}$ in a natural way via set inclusion. The collection of leaves from all trees is $K_{\sigma_{\nu}}$. From the construction it follows that all trees, $T(Q)$, with root cubes $Q$ from $\mathcal{F}^{(j)}(m)$ are isomorphic. 
We now consider the chosen minimizing cover, $\mathcal{F}$, and the corresponding family $\mathcal{F}^{(j)}(m)$. For each $Q \in K_{m}$, let $\mathcal{F}(Q)$ consist of the cubes in $\mathcal{F}$ that are subcubes to $Q$. We claim that the sums $\sum_{Q_{i} \in \mathcal{F}(Q)}\left|Q_{i}\right|^{r}$ have the same value for all $Q \in \mathcal{F}^{(j)}(m)$. Assume otherwise; then there are cubes $Q_{1}, Q_{2} \in \mathcal{F}^{(j)}(m)$ such that

$$
\sum_{Q_{i} \in \mathcal{F}\left(Q_{1}\right)}\left|Q_{i}\right|^{r}<\sum_{Q_{i} \in \mathcal{F}\left(Q_{2}\right)}\left|Q_{i}\right|^{r} .
$$

Since $\mathcal{F}$ is a cover of $K$, the Lemma shows that $\mathcal{F}$ also covers $K_{\sigma_{\nu}}$. The elements in $K_{\sigma_{\nu}}$ are the leaves in the trees $\left\{T(Q): Q \in K_{m}\right\}$. Hence, for each $Q \in K_{m}$, the leaves in $T(Q)$ are covered by $\mathcal{F}$. This implies that the unique path from each leaf in $T(Q)$ to the root node must contain a node in $\mathcal{F}$. Another way of saying this is that $\mathcal{F}(Q)$ covers the leaves in $T(Q)$.

By the isomorphism $\mathcal{I}$ from $T\left(Q_{1}\right)$ to $T\left(Q_{2}\right)$, the nodes $\mathcal{F}\left(Q_{1}\right)$ in $T\left(Q_{1}\right)$ correspond to some nodes, $\mathcal{I}\left(\mathcal{F}\left(Q_{1}\right)\right)$, in $T\left(Q_{2}\right)$. Thus, the unique path from each leaf in $T\left(Q_{2}\right)$ to the root node must contain a node in $\mathcal{I}\left(\mathcal{F}\left(Q_{1}\right)\right)$. Hence, $\mathcal{I}\left(\mathcal{F}\left(Q_{1}\right)\right)$ covers the leaves in $T\left(Q_{2}\right)$.

Now, if we remove the cubes in $\mathcal{F}\left(Q_{2}\right)$ from $\mathcal{F}$, and replace them with those in $\mathcal{I}\left(\mathcal{F}\left(Q_{1}\right)\right)$, we get a new collection of cubes, $\mathcal{F}^{\prime}$. From the reasoning above, it is clear that $\mathcal{F}^{\prime}$ also covers the leaves $K_{\sigma_{\nu}}$. Hence, by the Lemma, $\mathcal{F}^{\prime}$ is a cover of $K$. The Carathéodory sum of $\mathcal{F}^{\prime}$ is smaller than that of $\mathcal{F}$, because in the former, the partial sum $\sum_{Q_{i} \in \mathcal{F}\left(Q_{2}\right)}\left|Q_{i}\right|^{r}$ has been replaced with $\sum_{Q_{i} \in \mathcal{I}\left(\mathcal{F}\left(Q_{1}\right)\right)}\left|Q_{i}\right|^{r}$, which is smaller according to (15).

This contradicts the minimizing property of $\mathcal{F}$. Hence, the sum $\sum_{Q_{i} \in \mathcal{F}(Q)}\left|Q_{i}\right|^{r}$ is the same for all $Q \in \mathcal{F}^{(j)}(m)$. Moreover, this value must be $\left|Q_{0}\right|^{r}$, since $\mathcal{F}\left(Q_{0}\right)=\left\{Q_{0}\right\}$. This makes it possible to estimate the Carathéodory sum of $\mathcal{F}$ with respect to $r$ in a simple way.

Since the number of cubes in $\mathcal{F}^{(j)}(m)$ is $2^{\bar{\alpha}^{(j)}(m) \cdot m}$, it follows that

$$
\sum_{Q \in \mathcal{F}}|Q|^{r} \geq \sum_{Q \in \mathcal{F}(j)} \sum_{(m)}\left|Q_{i}\right|^{r}=\sum_{Q \in \mathcal{F}(Q)}\left|Q_{0}\right|^{r} \geq 2^{\bar{\alpha}^{(j)}(m)}(m) \cdot 2^{-m r} .
$$

From (8) and (11), it follows that $\bar{\alpha}^{(j)}(m) \geq r-n / m$, and using this we get

$$
\sum_{Q \in \mathcal{F}}|Q|^{r} \geq 2^{(r-n / m) m} 2^{-m r}=2^{-n} .
$$

Since $\mathcal{F}$ is a cover that minimizes the Carathéodory sum with respect to $r$, this shows that the lower bound $2^{-n}$ is valid for all covers under consideration, i.e. covers consisting of a collection of cubes in our construction with side lengths in $\left(2^{-\sigma_{\nu}}, 2^{-\sigma_{p}}\right]$. Moreover, the lower bound $2^{-n}$ does not depend on $p$ or $\nu$. Hence, by letting $\nu \rightarrow \infty$ and then $p \rightarrow \infty$, we get $H^{r}(K) \geq 2^{-n}$, and thereby $\operatorname{dim}_{\mathrm{H}}(K) \geq r$. 
We now show the upper estimate $\operatorname{dim}_{H}(K) \leq r$. Consider a cover of $K$ consisting of the cubes in

$$
\mathcal{F}=\mathcal{F}^{(1)}\left(i_{2}\right) \cup \mathcal{F}^{(0)}\left(i_{5}\right),
$$

in Multistep $p+1$. The number of cubes in this cover, $\# \mathcal{F}$, can be estimated with (8) and (11) as

$$
\# \mathcal{F}^{(1)}\left(i_{2}\right)=2^{\bar{\alpha}^{(1)}\left(i_{2}\right) \cdot i_{2}}<2^{r i_{2}}, \quad \# \mathcal{F}^{(0)}\left(i_{5}\right)=2^{\bar{\alpha}^{(0)}\left(i_{5}\right) \cdot i_{5}}<2^{r i_{5}} .
$$

This gives us

$$
\sum_{Q \in \mathcal{F}}|Q|^{r}<2^{r i_{2}} \cdot\left(\sqrt{n} 2^{-i_{2}}\right)^{r}+2^{r i_{5}} \cdot\left(\sqrt{n} 2^{-i_{5}}\right)^{r}=2 n^{r / 2}
$$

Letting $p \rightarrow \infty$ gives us $H^{r}(K) \leq 2 n^{r / 2}$, so $\operatorname{dim}_{\mathrm{H}}(K) \leq r$.

This proves $\operatorname{dim}_{\mathrm{H}}(U)=r$ if $U=K$. Now, consider an arbitrary nonempty set $U$ that is relatively open in $K$, i.e. $U=O \cap K$ for some $O$ open in $\mathbb{R}^{n}$. Since $O$ is open, there must exist a cube $Q^{*} \subset O$ that belongs to $\mathcal{F}^{(j)}\left(\sigma_{p}\right)$ for some $j \in\{0,1\}$ and $p \geq 1$. All cubes in $\mathcal{F}=\mathcal{F}^{(0)}\left(\sigma_{p}\right) \cup \mathcal{F}^{(1)}\left(\sigma_{p}\right)$ have the same future, in the sense that they have the same number of subcubes in the subsequent families. This implies that $\operatorname{dim}_{\mathrm{H}}\left(Q_{1} \cap K\right)=\operatorname{dim}_{\mathrm{H}}\left(Q_{2} \cap K\right)$ for all $Q_{1}, Q_{2} \in \mathcal{F}$. This gives

$$
\begin{aligned}
\operatorname{dim}_{\mathrm{H}}(K) & =\operatorname{dim}_{\mathrm{H}}\left(\bigcup_{Q \in \mathcal{F}}(Q \cap K)\right)=\max \left\{\operatorname{dim}_{\mathrm{H}}(Q \cap K): Q \in \mathcal{F}\right\} \\
& =\operatorname{dim}_{\mathrm{H}}\left(Q^{*} \cap K\right) \leq \operatorname{dim}_{\mathrm{H}}(O \cap K) \\
& \leq \operatorname{dim}_{\mathrm{H}}(K) .
\end{aligned}
$$

Hence, since $U=O \cap K$ and we already know that $\operatorname{dim}_{H}(K)=r$, we have $\operatorname{dim}_{H}(U)=r$. This concludes the proof of the theorem.

\section{References}

[1] K. Falconer, Fractal Geometry. Mathematical Foundations and Applications, Wiley, Chichester, 1990.

[2] S. P. Lalley and D. Gatzouras, Hausdorff and box dimensions of certain self-affine fractals, Indiana Univ. Math. J. 41 (1992), 533-568.

[3] C. McMullen, The Hausdorff dimension of general Sierpiński carpets, Nagoya Math. J. 96 (1984), 1-9.

[4] Y. Pesin and H. Weiss, On the dimension of deterministic and random Cantor-like sets, symbolic dynamics, and the Eckmann-Ruelle conjecture, Comm. Math. Phys. 182 (1996), 105-153. 
[5] D. W. Spear, Sets with different dimensions in [0,1], Real Anal. Exchange 24 (1998/99), 373-389.

Department of Mathematics and Mathematical Statistics

Umeå University

90187 Umeå, Sweden

E-mail: anders.nilsson@math.umu.se

peter.wingren@math.umu.se

Received June 8, 2006

Revised version June 18, 2007 